\title{
The photonic wheel - demonstration of a state of light with purely transverse angular momentum
}

\author{
P. Banzer \\ peter.banzer@mpl.mpg.de
}

\section{Neugebauer}

\section{A. Aiello}

\section{Marquardt}

\section{N. Lindlein}

\section{T. Bauer}

\section{G. Leuchs}

Max Planck Institute for the Science of Light, Guenther-Scharowsky-Str. 1, 91058 Erlangen, Germany Institute of Optics, Information and Photonics, University Erlangen-Nuremberg, Staudtstr. 7/B2, 91058 Erlangen, Germany

Max Planck Institute for the Science of Light, Guenther-Scharowsky-Str. 1, 91058 Erlangen, Germany Institute of Optics, Information and Photonics, University Erlangen-Nuremberg, Staudtstr. 7/B2, 91058 Erlangen, Germany

Max Planck Institute for the Science of Light, Guenther-Scharowsky-Str. 1, 91058 Erlangen, Germany Institute of Optics, Information and Photonics, University Erlangen-Nuremberg, Staudtstr. 7/B2, 91058 Erlangen, Germany

Max Planck Institute for the Science of Light, Guenther-Scharowsky-Str. 1, 91058 Erlangen, Germany Institute of Optics, Information and Photonics, University Erlangen-Nuremberg, Staudtstr. 7/B2, 91058 Erlangen, Germany

Institute of Optics, Information and Photonics, University Erlangen-Nuremberg, Staudtstr. 7/B2, 91058 Erlangen, Germany

Max Planck Institute for the Science of Light, Guenther-Scharowsky-Str. 1, 91058 Erlangen, Germany Institute of Optics, Information and Photonics, University Erlangen-Nuremberg, Staudtstr. 7/B2, 91058 Erlangen, Germany

Max Planck Institute for the Science of Light, Guenther-Scharowsky-Str. 1, 91058 Erlangen, Germany Institute of Optics, Information and Photonics, University Erlangen-Nuremberg, Staudtstr. 7/B2, 91058 Erlangen, Germany

In classical mechanics, a system may possess angular momentum which can be either transverse (e.g. in a spinning wheel) or longitudinal (e.g. for a spiraling seed falling from a tree) with respect to the direction of motion. However, for light, a typical massless wave system, the situation is less versatile. Photons are well-known to exhibit intrinsic angular momentum which is longitudinal only: the spin angular momentum defining the polarization and the orbital angular momentum associated with a spiraling phase front. Here we show that it is possible to generate a novel state of the light field that contains purely transverse angular momentum, the analogue of a spinning mechanical wheel. We realize this state by tight focusing of a polarization tailored light beam and measure it using an optical nano-probing technique. Such a novel state of the light field can find applications in optical tweezers and spanners where it allows for additional rotational degree of freedom not achievable in single-beam configurations so far.

[DOI: http://dx.doi.org/10.2971/jeos.2013.13032]

Keywords: Novel state of the light field, transverse angular momentum, tight focusing, nano-probing

\section{INTRODUCTION}

Spinning objects such as the wheels of a running bicycle or propellers driving aircraft or ships share an important property: they display a rotatory motion quantifiable via the angular momentum (AM) J. The latter is purely transverse for a rolling wheel that rotates around an axis perpendicular to the direction of motion of the bicycle, while it is entirely longitudinal for a spiraling seed [1] or a propeller which rotates around an axis parallel to the direction of motion. These examples show that the AM of a massive system can be either transverse or longitudinal to the direction of motion of the system itself.

However, when considering a massless wave system, such as light, the possibilities are more limited. Sophisticated group theory arguments show that a photon in a plane wave state with an exactly determined linear momentum $\mathbf{p}$ can only possess a longitudinal AM either parallel or antiparallel to $\mathbf{p}[2,3]$. In fact, photons may exhibit both a longitudinal spin AM (the so-called helicity) [4], that defines the polarization of the light, and a longitudinal orbital AM [5]-[7], that sets the vorticity of the light. Thus, as far as the behavior with respect to AM is concerned, light is dynamically closer to a falling seed rather than a bicycle wheel. At the same time, there is not a no-go theorem that forbids the existence of more general states of light with purely transverse AM. Nevertheless, the existence of a photonic state analogue to a rolling wheel has eluded theoretical studies and especially experimental verification up to now. Furthermore, it allows for additional rotational degree of free- 


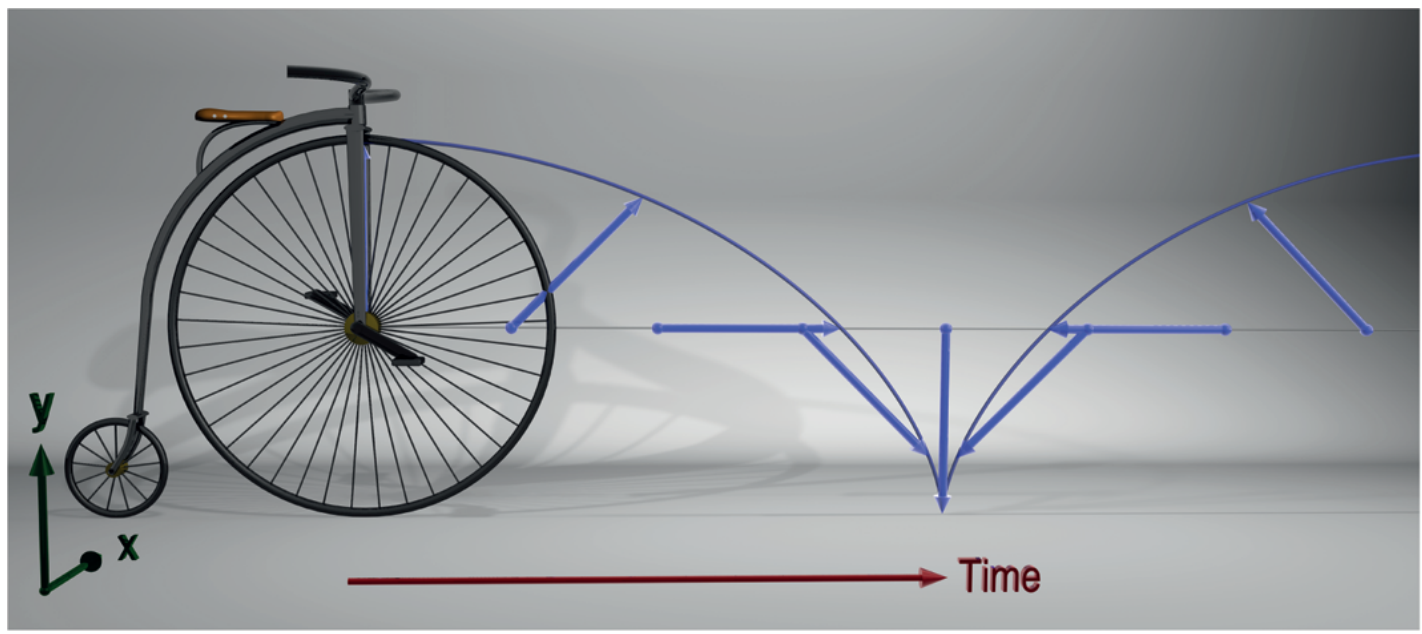

FIG. 1 Artistic illustration of a photonic wheel in analogy to a rolling mechanical wheel. The horizontal axis represents the time. The $x$ - and $y$-axis span the focal plane for $z=0$. The end tip of a single spoke of the bicycle's wheel traces a cycloidal curve in time. The same holds true for the electric field vector in a photonic wheel, which spins around the transverse coordinate axis $x$ in the focal plane with time. The shown arrows represent the yz-components of the electric field vector at defined positions in the focal plane for a state of light with purely transverse AM.

dom in optical tweezers. A particle trapped in such a highly confined state of the light field would rotate around a transverse axis perpendicular to the optical axis. Such a scheme was not realized so far in single beam configurations [8]-[11].

In our studies, we use an optical nano-probing technique $([12,13]$; see also Appendix 6.1) to demonstrate the occurrence of a state of light possessing purely transverse AM, namely a "photonic wheel," (see Figure 1), in the focal plane of a high numerical aperture (NA) focusing system. The appearance of this novel state of light can be seen in connection with the recently discovered spin Hall effect of light (SHEL) [14]-[19] implied by transverse AM. In our setup, a linearly polarized input beam of light is first converted into a laterally segmented beam consisting of two parts with opposite spin direction ( $\sigma=-1$, left-circularly polarized and $\sigma=+1$, right-circularly polarized) using a special optical element. The resulting beam is then tightly focused by a microscope objective (see Figure 2(a) and (b)). This yields to the occurrence of a purely transverse AM in the focal plane of the objective, as schematically illustrated in Figure 2(b). We note here that similar experimental schemes were proposed by [20]-[22], but used for very different types of measurements and studies.

\section{THEORY}

The electric field of a beam of light in the focus of a lens can be calculated by using the standard vectorial diffraction theory [23]. For that purpose, the input beam is sampled on a grid of points in the entrance pupil of the focusing system. At each sampling point the beam is locally treated as a geometrical ray being refracted by the lens system taking into account its state of polarization and propagating towards the geometrical focus with an individual k-vector. In the focal plane, these rays represent local plane wave components which interfere with each other forming the focal field distribution. The corresponding integrals are solved numerically. A total number of $200 \times 200$ plane waves were used for the decomposition of the input beam. The presented area of the focal plane of
$2 \mu \mathrm{m} \times 2 \mu \mathrm{m}$ was sampled with a total number of $400 \times 400$ data points.

The results of these calculations for our laterally split input beam are depicted in Figure 3(a)-(d) for the energy densities of the individual field components and for the total electric energy density. It may be seen that due to interference of the two halves of the beam with different spin, the distributions of the electric field $y$ - and $z$-components show a pronounced shift in positive $y$-direction in the focal plane with the $y$-axis being the symmetry axis of the observed patterns. At the same time, the x-component of the electric field is shifted in the opposite direction. All electric field components are of comparable strength. Moreover, the $y$ - and $z$-components are $\pi / 2$ out of phase leading to an electric field spinning around the transverse x-axis, as shown in Figure 1. In contrast, the electric field is linearly polarized in $x$-direction on the optical axis of the focusing system $(x=y=0)$. By changing the relative phase between the two segments of the input beam the spinning electric field can be moved towards the optical axis in the focal plane (see results below).

Finally, the total electric field energy density distribution $\left|\mathbf{E}_{t o t}\right|^{2}$ exhibits a distinct shape in the focal plane (see Figure 3(d)). Furthermore, its barycenter is shifted in positive ydirection which is a result of interference in the focal plane and can be seen as a manifestation of the geometric SHEL [18, 19] in the given scheme due to the transverse component $\mathrm{J}_{x}$ of the beam AM. Concurrently, no spiraling phase front and thus no longitudinal OAM of one of the electric field components is observed in the focal plane (see insets in Figure 3(a)-(c)) which would be typical for a homogeneously circularly polarized beam that undergoes spin-to-orbital AM conversion by focusing [24]-[26]. The cancellation of the longitudinal AM in the focal plane is pictorially illustrated in Figure 2(b) and calculated in Figure 3(e)-(g) where the focal distributions of the components of the AM density $\mathbf{j}=\left(\mathbf{j}_{x}, \mathbf{j}_{y}, \mathbf{j}_{z}\right)$ are shown. Please note, that the distribution of the $z$-component of the AM density is strictly zero everywhere in the focal plane while it is non-zero in the two segments of the input beam 


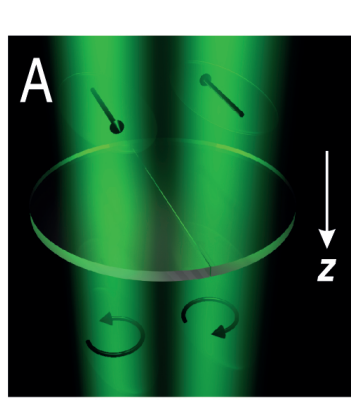

B

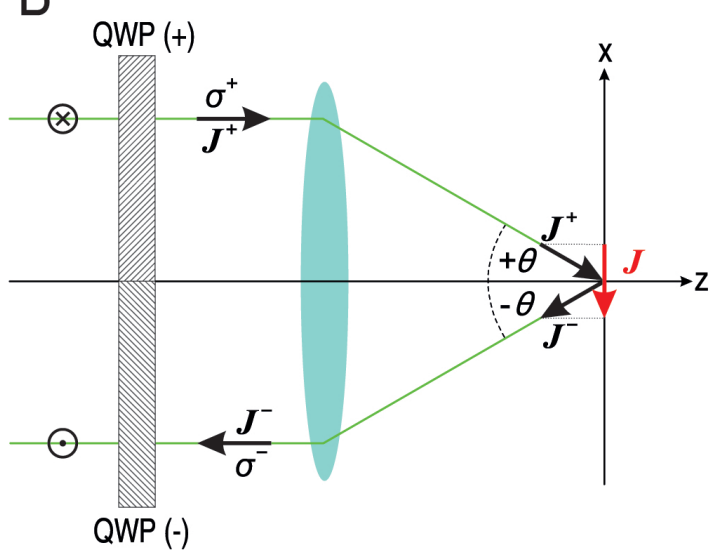

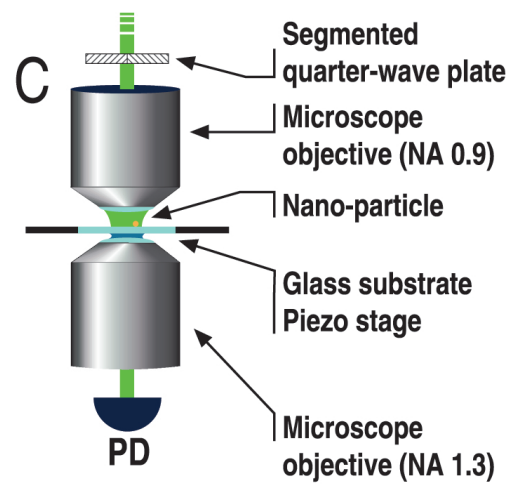

bjective (NA 1.3)
FIG. 2 Generation of a "photonic wheel". (A) A linearly polarized input beam (here a ypolarized TEM10 mode) is transformed into a split beam with separated left- and righthanded circular polarization using a segmented quarter-wave plate. (B) When tightly focused, the longitudinal components of $\mathrm{J}^{+}$and $\mathrm{J}^{-}$cancel, while their transverse components add up to a purely transverse AM (red) in the focal plane. (C) The state of purely transverse AM is generated in the focus of a microscope objective with an NA of 0.9. A gold nano-particle (diameter $90 \mathrm{~nm}$ ) sitting on a glass substrate is scanned through the focal plane. For every position of the particle in the beam the forward scattered and transmitted light is collected by an immersion-type microscope objective $(\mathrm{NA}=1.3)$ and detected with a single photodiode.

in front of the focusing lens. By integrating the AM density distributions over the focal plane, the AM per unit length $\mathbf{J}=\iint \mathbf{j} \mathrm{d} x \mathrm{dy}=\left(\mathrm{J}_{x}, \mathrm{~J}_{y}, \mathrm{~J}_{z}\right)$ can be obtained. It follows that $\mathrm{J}_{y}=\mathrm{J}_{z}=0$ while $\mathrm{J}_{x} \neq 0$ because $\mathrm{j}_{x}$ exhibits an asymmetric distribution along the y-axis (see Figure 3(h)). This theoretically demonstrates that the AM is purely transverse in the focal plane, thus revealing the existence of a novel state of light, the "photonic wheel". It is important to note here that we can actually realize such a beam (with $\mathrm{J}_{z}=0$ while e.g. $\mathrm{J}_{x} \neq 0$ ) not only in the focal plane. When leaving the focal plane, the longitudinal AM density $\mathrm{j}_{z}$ is not zero anymore but presents an anti-symmetric distribution that after integration results in a vanishing $\mathrm{J}_{z}$.

\section{EXPERIMENT AND RESULTS}

In the experimental setup the light beam from a supercontinuum light-source is spectrally filtered using an acousto-optical tunable spectral filter operating in the visible spectral range. Thereupon, the beam is coupled into a single-mode fiber for mode-cleaning purposes. Behind the fiber the linearly polarized Gaussian-shaped beam is collimated again. In our experiments, a special optical ele- ment (custom-built by B. Halle Nachfl. GmbH) is utilized to generate the tailored polarization and spin distribution which is then focused tightly (see Figure 2(a) and (b)). The element consists of two identical quarter-wave plates, merged with their fast-axes being oriented under $+45^{\circ}$ and $-45^{\circ}$ with respect to the bonding axis. With an appropriate choice of the polarization direction of the linearly polarized input beam, the desired beam is generated with spatially separated left- and right-handed circular polarization. If the input beam is converted to a Hermite-Gaussian mode of odd order (for instance a linearly polarized $\mathrm{TEM}_{10}$ mode) before passing through the segmented wave plate, diffraction originating from the split region between both wave plates can be drastically reduced. Furthermore, the maxima of the intensity distribution of the input field are shifted towards the outer rim of the entrance aperture. Therefore, on the one hand stronger longitudinal field components are generated in the focal plane relative to the transverse field components. On the other hand, also stronger transverse AM is created in the focal plane leading to a more pronounced shift of the barycenter of the electric energy density distribution. The theoretical as well as experimental results presented in this article were achieved using such a linearly polarized $\mathrm{TEM}_{10}$ mode as an input beam for the segmented quarter-wave plate. The resulting optical beam is then tightly focused by a microscope objective with an NA of 0.9 (see Figure 2(b)) generating the aforementioned "photonic wheel". We use a scanning technique employing a single nano-particle on a substrate as a field-probe (see Figure 2(c)) to experimentally proof the aforementioned statements about the focal shape of $\left|\mathbf{E}_{t o t}\right|^{2}$ for the generated state of light with purely transverse AM (see Appendix 6.2). The sample consists of single and well separated spherical nano-particles made from gold with a diameter of approximately $90 \mathrm{~nm}$ sitting on a glass substrate (see Appendix 6.1), which can be positioned relative to the focal plane with nanometer resolution using a 3D piezostage. In the experiment, the single nano-particle is scanned through the beam in the focal plane (see Figure 2(c)). For every position of the nano-particle relative to the beam, the sub-wavelength particle interacts with the local electric field only. By detecting the light being scattered by the particle as a function of its position relative to the focused beam in the focal plane, we are able to probe the focal distribution of $\left|\mathbf{E}_{t o t}\right|^{2}$ (see Appendix 6.2). In the setup the forward-scattered and transmitted light is collected with an immersion-type microscope objective with an NA of 1.3 and guided onto a photodiode (see Figure 2(c)). For the measurements we choose a wavelength of $530 \mathrm{~nm}$ close to the plasmonic particle resonance. The employed experimental setup is similar to the one we already described in [27].

In Figure 4(a) we show our experimental scan result for the helicity tailored input beam measured in transmission. A drop in signal strength is observed, when the particle is overlapping with the beam in the focal plane. A smile-like deformation is observed experimentally for the specially tailored input polarization distribution described above. For comparison, the theoretical distribution of $\left|\mathbf{E}_{t o t}\right|^{2}$ calculated for the experimental parameters is presented again in Figure 4(b). Please note that the same data was already shown in Figure 3(d). For better 

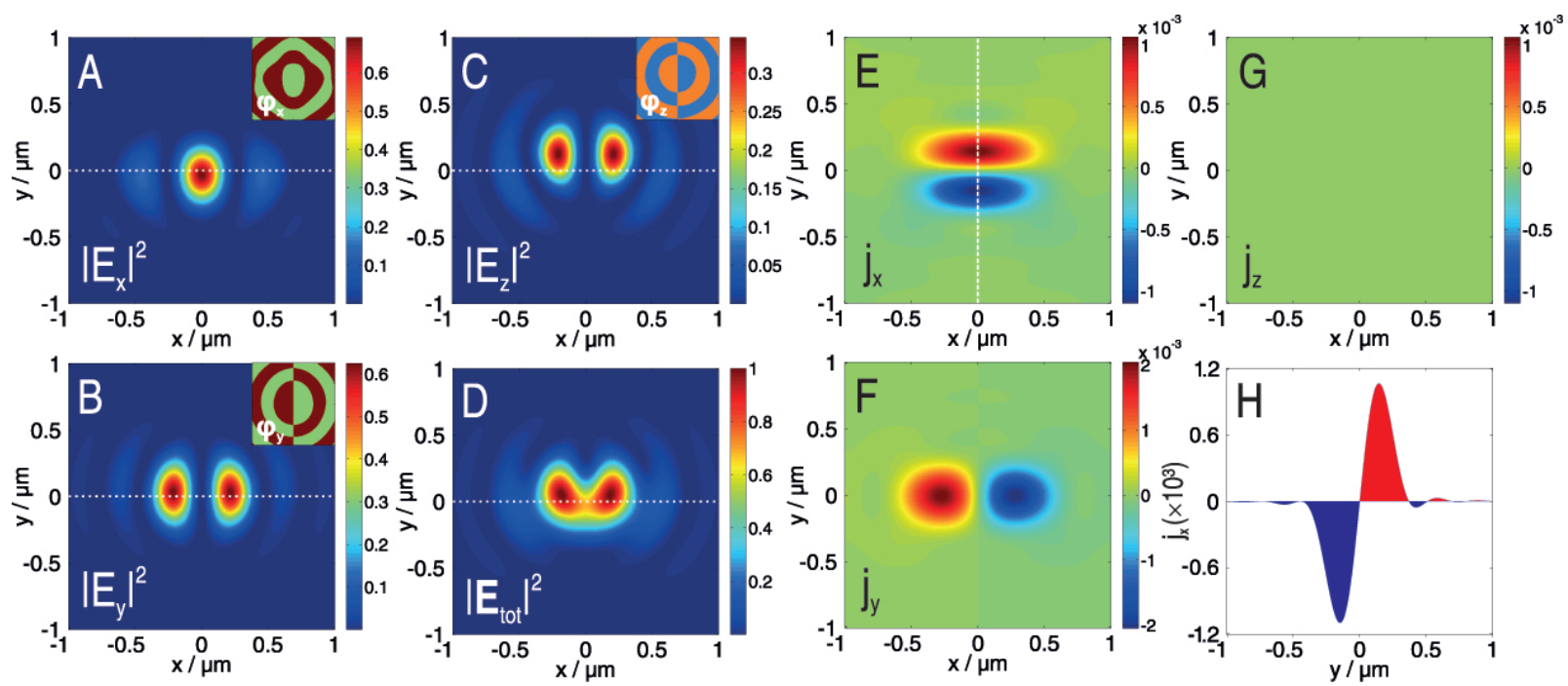

FIG. 3 Calculated electric energy and angular momentum density components in the focal plane. (A) - (D) Theoretical distributions of $\left|\mathbf{E}_{x}\right|^{2}$, $\left|\mathbf{E}_{y}\right|^{2}$, $\left|\mathbf{E}_{z}\right|^{2}$ and $\left|\mathbf{E}_{\text {tot }}\right|^{2}$ for a helicity tailored input beam (see Figure $2(\mathrm{a})$ ) in the focal plane (normalized to $\max \left(\left|\mathbf{E}_{t o t}\right|^{2}\right)$ ). Insets: corresponding distributions of the relative phases $\varphi_{x}, \varphi_{y}$ and $\varphi_{z}($ dark red: $\pi$; light green: 0 ; orange: $+\pi / 2$; light blue: $-\pi / 2$ ). (E) - (G) Calculated distributions of the components $j_{x}, j_{y}$ and $j_{z}$ of the AM density $j$ confirming the existence of purely transverse AM in the focal plane. (H) Cut through the asymmetric distribution of $j_{x}$ along the $y$-axis as indicated by the dashed white line in (E).

comparability with the experiment we use an inverted colorscale here. The experimentally measured shape of the beam in the focus is in excellent agreement with our theoretical predictions. Slight asymmetries in the experimental result are caused by deviations of the particle shape from an ideal sphere and by imperfections of the split-aperture etc. From a theoretical point of view, the experimentally measured shape in the focal plane (Figure 4(a)) in combination with the chosen input polarization state is directly connected to the fact that the beam is in a state of purely transverse AM in the investigated plane $\left(\mathrm{J}_{z}=0\right)$.

For practical application of the presented scheme, the freedom of changing the focal electric energy density distribution while preserving the purely transverse character of the AM can be of great importance. Therefore, we also investigated a second input field distribution which generates a state of purely transverse AM in the focus, but exhibits a fundamentally different distribution of $\left|\mathbf{E}_{t o t}\right|^{2}$ and its components. For that purpose, the relative phase between the leftand right-handed circular polarizations in the two beam segments of the input beam is changed (additional phase shift of $\pi$ ). The investigated input distribution is presented in Figure 4(c) (left). The corresponding experimental result and the calculated total electric energy density distribution are shown in Figure 4(c) and (d). The focal distribution has changed drastically. This is because, for instance, the maximum electric energy density of the z-component of the electric field is now located on the y-axis also exhibiting a pronounced shift along this axis relative to the origin (not shown here). Furthermore, the maximum of the y-component of the electric energy density is found to lie on the $y$-axis as well. The $z$ - and $y$ component of the electric field are $\pi / 2$ out of phase, which is in line with the predicted purely transverse angular momentum. In addition, the $x$-component of the electric field has a zero crossing on the $y$-axis. In this configuration the distributions of the AM density components are equal to those already presented in Figure 3(e)-(h), thus still yielding $\mathrm{J}_{y}=\mathrm{J}_{z}=0$ and $\mathrm{J}_{x} \neq 0$.

In addition to the presented experimental results for photonic wheels, we confirm that the experimentally observed shape of the beams in the focal plane is not caused by any aberration of our optical system but is indeed a direct consequence of the helicity distribution in the entrance aperture. For that purpose, we use a linearly (x-) polarized Gaussian input beam which is tightly focused. It is known that due to the vectorial character of the light field the focal distribution of $\left|\mathbf{E}_{t o t}\right|^{2}$ should be elongated along the axis of polarization $[23,28]$. For comparison, the corresponding theoretical distribution is presented in Figure 4(f). The experimental scan image is in good agreement with the theoretically predicted shape and the elongation along the x-axis is clearly visible (see Figure 4(e)).

\section{APPLICATION AND CONCLUSIONS}

We believe that such a novel state of the light field, a "photonic wheel", can find application in optical tweezers and in nano-manipulation setups. A trapped particle should rotate around a transverse axis in this scheme in contrast to conventional AM transfer resulting in a rotation around the longitudinal axis. When combined with spin and orbital AM in the focal plane [8]-[11], such an implementation paves the way to three-dimensional rotation control over a trapped particle in optical tweezers allowing for more freedom in particlemanipulation in, for instance, biological applications or in driving micro-devices.

\section{ACKNOWLEDGEMENTS}

We thank M. Schmelzeisen from the Max Planck Institute for Polymer Research in Mainz, Germany for fabricating the spherical nanoparticles used in our experiments. 


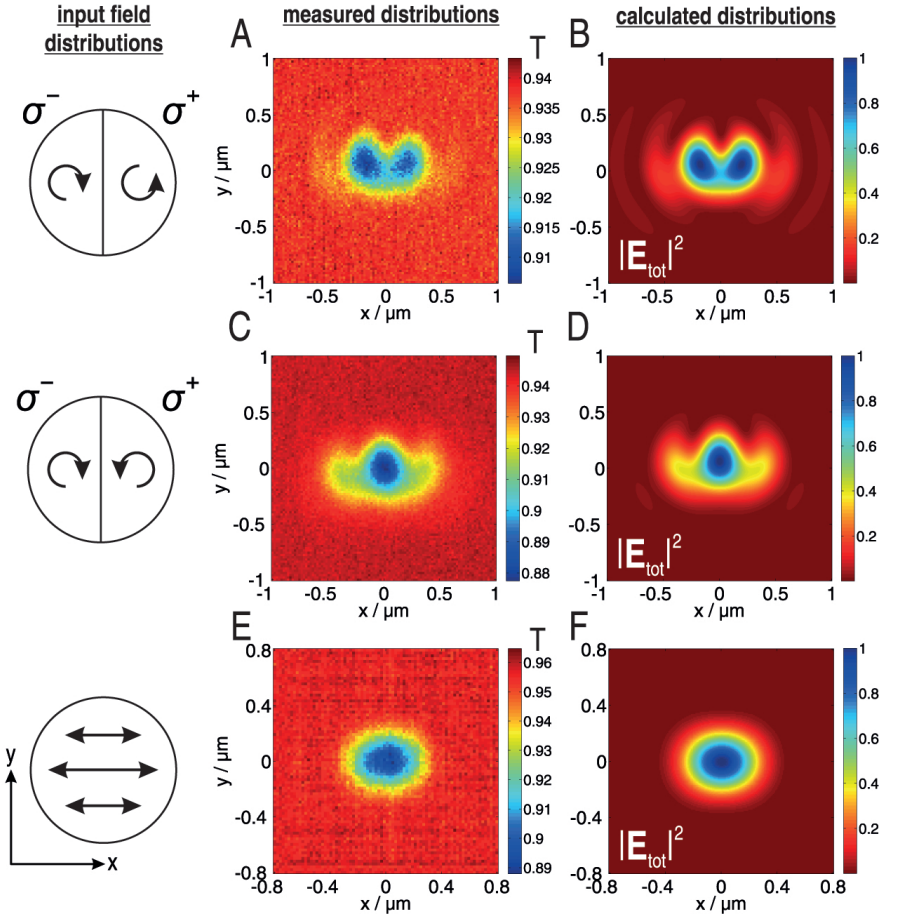

FIC. 4 Results. Experimental scan results (wavelength: $530 \mathrm{~nm}, \mathrm{NA}=0.9$ ) (A) for the "photonic wheel" resulting from tight focusing of a helicity tailored input beam, (C) for the same tailored beam but with an additional $\pi$ phase-shift between the segments and (E) for a tightly focused and linearly ( $\mathrm{x}$-) polarized input beam (see left panels). Every pixel of the presented 2D scan images corresponds to one position of the nanoparticle relative to the beam in the focal plane. The integrated transmitted and forward scattered intensity for each position is shown. The experimental data is normalized to the transmission of the focused beam through the glass substrate. (B), (D) and (F) show the corresponding calculated distributions of $\left|E_{t o t}\right|^{2}$ (shown with inverted color-map for better comparability).

\section{APPENDIX}

\subsection{Sample fabrication}

The spherical shape of the single gold nano-particles is achieved by treating conventional and commercially available gold nano-particles with intense laser pulses (see for instance [29]). This procedure was performed at the Max Planck Institute for Polymer Research (MPIP) in Mainz, Germany. The particles are molten by the high localized energy of the light pulses. Due to surface tension, the particles reform to an almost perfectly spherical shape. The nano-particles are dispersed in water and have a diameter of approximately 90 $\mathrm{nm}$. Marker structures are patterned on the glass substrate (BK7) using laser-lithography before the particles are spincoated. The marker structures are utilized for orientation and precise focus alignment purposes in the experiment. Furthermore they allow for an exact characterization of the employed nano-particle.

\subsection{Reconstruction of the focal electric energy density distribution using a single gold nano-particle on a substrate}

Let us consider the basic case of a point-like electric dipole oscillating harmonically in free space or inside a homoge- neous medium. It is well known that the emitted power has a $\sin ^{2}(\theta)$ distribution with $\theta$ being the angle relative to the dipole moment. By defining the $\mathrm{z}$-axis as the optical axis of an optical system collecting the emitted power, two special cases can be investigated: the dipole moment perpendicular (transverse) or parallel (longitudinal) to the z-axis. If in both cases the dipole moment is of the same strength, a full hemisphere along the positive or negative z-axis has to be collected to measure the same integrated power in both scenarios, which is half of the total power emitted. If instead a limited solid angle (NA) is used for collection, the integrated collected power will be mismatched. Equivalently, if for instance a single spherical nano-particle embedded in free-space or a homogeneous medium is used to interact with the electric energy density distribution in the focal plane of a high NA optical system, the same considerations have to be taken into account. If the particle is chosen to be sufficiently small, its dipolar response dominates. Here, the nano-particle is excited by the local electric field, which is almost homogeneous across the particle diameter. The direction of the induced electric dipole moment then just depends on the direction of the locally linear electric field it is overlapping with. When collecting the forward scattered and transmitted and the backwardscattered light in this configuration, a mismatch between the collected integrated power is observed for a longitudinally or transversely oscillating electric dipole which was induced in the particle with equal strength. Furthermore, in forward direction also the transmitted part of the incoming beam which has not interacted with the particle and which can interfere with the forward-scattered part of the light field is present. If in a next step either the electric point-like dipoles or the scattering particle are located close to a dielectric interface the situation changes significantly. For instance, as a consequence of the glass substrate used as a support for the nano-particle in the actual experiment, its resonance is spectrally red-shifted relative to the free-space case, which is taken into account in the experiments by choosing a proper wavelength of excitation. Beside the red-shift of the spectral resonance position induced by the presence of a dielectric interface, also the emission pattern of a point-like electric dipole or the excited nanoparticle is changed drastically. Although the particle is small in diameter and the excitation is locally linear, the emission is not purely dipole-like anymore and the emitted pattern in the glass hemisphere differs from that in air [30]-[32]. Hence, the integrated emissions into the air and glass hemisphere are not equal in this case. It can be shown, that in such a situation collecting the forward scattered light within a solid angle of less than $2 \pi$ is sufficient to measure the same power emitted by electric dipoles excited in the particle by equally strong longitudinally or transversely oscillating electric fields. Thus, the energy densities of the corresponding field components can be reconstructed correctly by using a nano-particle as a field probe. Furthermore, the transmitted light which has not interacted with the particle and which can interfere with the forward scattered light changes the angular dependence slightly. In the experimental system we used for the measurements the transmitted and forward scattered light is collected with a microscope objective with an NA of 1.3. The corresponding collection angle within the glass hemisphere is larger than the angle discussed above for which longitudinal and transverse field components are probed equally. Nevertheless, only 
a slight mismatch between the reconstructed longitudinal (z) and transverse ( $\mathrm{x}$ and $\mathrm{y}$ ) components of the electric energy density is expected. Thus, for confirming the deformed shape of our beam in the focal plane, the chosen and simplified scheme of measurement is sufficient. It is worth mentioning here that the highly accurate reconstruction of amplitude and even phase of the individual electric field components of tightly focused and arbitrarily polarized beams by taking into account the aforementioned details will be reported elsewhere soon.

\section{References}

[1] A. Azuma, and K. Yasuda, "Flight Performance of Rotary Seeds," J. Theor. Biol. 138, 23-53 (1989).

[2] E. P. Wigner, "On the unitary representations of the inhomogeneous Lorentz group," Ann. Math. 40, 149-204 (1939).

[3] T. D. Newton, and E. P. Wigner, "Localized States for Elementary Systems," Rev. Mod. Phys. 21, 400-406 (1949).

[4] L. Mandel, and E. Wolf, Optical coherence and quantum optics (Cambridge University Press, Cambridge, 1995).

[5] L. Allen, M. W. Beijersbergen, R. J. C. Spreeuw, and J. P. Woerdman, "Orbital angular momentum of light and the transformation of Laguerre-Gaussian laser modes," Phys. Rev. A 45, 8185-8189 (1992).

[6] A. M. Yao, and M. J. Padgett, "Orbital angular momentum: origins, behavior and applications," Advances in Optics and Photonics 3, 161-204 (2011).

[7] J. F. Nye, and M. V. Berry, "Dislocations in wave trains," P. R. Soc. A 336, 165-190 (1974).

[8] J. Poynting, "The wave motion of a revolving shaft, and a suggestion as to the angular momentum in a beam of circularly polarised light," P. R. Soc. Lond. A 82, 560-567 (1909).

[9] R. Beth, "Mechanical detection and measurement of the angular momentum of light," Phys. Rev. 50, 115-125 (1936).

[10] H. He, M. E. J. Friese, N. R. Heckenberg, and H. Rubinsztein-Dunlop, "Direct Observation of Transfer of Angular Momentum to Absorptive Particles from a Laser Beam with a Phase Singularity," Phys. Rev. Lett. 75, 826-829 (1995).

[11] M. Padgett, and R. Bowman, "Tweezers with a twist," Nat. Photonics 5, 343-348 (2011).

[12] T. Züchner, A. V. Failla, A. Hartschuh, and A. J. Meixner, “A novel approach to detect and characterize the scattering patterns of single Au nanoparticles using confocal microscopy," J. Microsc. 229, 337-342 (2008).

[13] 0. G. Rodriguez-Herrera, D. Lara, K. Y. Bliokh, E. A. Ostrovskaya, and C. Dainty, "Optical Nanoprobing via Spin-Orbit Interaction of Light," Phys. Rev. Lett. 104, 253601 (2010).

[14] M. Onoda, S. Murakami, and N. Nagaosa, "Hall Effect of Light," Phys. Rev. Lett. 93, 083901 (2004).

[15] K. Y. Bliokh, and Y. P. Bliokh, "Conservation of Angular Momentum, Transverse Shift, and Spin Hall Effect in Reflection and Refraction of an Electromagnetic Wave Packet," Phys. Rev. Lett. 96, 073903 (2006).
[16] K. Y. Bliokh, and Y. P. Bliokh, "Polarization, transverse shifts, and angular momentum conservation laws in partial reflection and refraction of an electromagnetic wave packet," Phys. Rev. E 75 , 066609 (2007).

[17] 0. Hosten, and P. Kwiat, "Observation of the Spin Hall Effect of Light via Weak Measurements," Science 319, 787-790 (2008).

[18] A. Aiello, N. Lindlein, C. Marquardt, and G. Leuchs, "Transverse Angular Momentum and Ceometric Spin Hall Effect of Light," Phys. Rev. Lett. 103, 100401 (2009).

[19] J. Korger, A. Aiello, C. Gabriel, P. Banzer, T. Kolb, C. Marquardt, and G. Leuchs, "Geometric Spin Hall Effect of Light at polarizing interfaces," Appl. Phys. B 102, 427-432 (2011).

[20] N. B. Baranova, A. Y. Savchenko, and B. Y. Zel'dovich, "Transverse shift of a focal spot due to switching of the sign of circular polarization," JETP Lett. 59, 232-234 (1994).

[21] B. Y. Zel'dovich, N. D. Kundikova, and L. F. Rogacheva, "Observed transverse shift of a focal spot upon a change in the sign of circular polarization," JETP Lett. 59, 766-769 (1994).

[22] Z. Bomzon, and M. Gu, "Space-variant geometrical phases in focused cylindrical light beams," Opt. Lett. 32, 3017-3019 (2007).

[23] B. Richards, and E. Wolf, "Electromagnetic diffraction in optical systems, II. Structure of the image field in an aplanatic system," P. R. Soc. Lond. A 253, 358-379 (1959).

[24] Y. Zhao, J. S. Edgar, G. D. M. Jeffries, D. McGloin, and D. T. Chiu, "Spin-to-Orbital Angular Momentum Conversion in a Strongly Focused Optical Beam," Phys. Rev. Lett. 99, 073901 (2007).

[25] T. A. Nieminen, A. B. Stilgoe, N. R. Heckenberg, and H. RubinszteinDunlop, "Angular momentum of a strongly focused Gaussian beam," J. Opt. A: Pure Appl. Opt. 10, 115005-1-6 (2008).

[26] K. Y. Bliokh, M. A. Alonso, E. A. Ostrovskaya, and A. Aiello, “Angular momenta and spin-orbit interaction of nonparaxial light in free space," Phys. Rev. A 82, 063825-1-7 (2010).

[27] P. Banzer, U. Peschel, S. Quabis, and G. Leuchs, "On the experimental investigation of the electric and magnetic response of a single nano-structure," Opt. Express 18, 10905-10923 (2010).

[28] R. Dorn, S. Quabis, and G. Leuchs, "The focus of light - linear polarization breaks the rotational symmetry of the focal spot," J. Mod. Opt. 50, 1917-1926 (2003).

[29] S. Link, and M. A. El-Sayed, "Spectroscopic determination of the melting energy of a gold nanorod," J. Chem. Phys. 114, 2362 (2001).

[30] J.-Y. Courtois, J.-M. Courty, J. C. Mertz, "Internal dynamics of multilevel atoms near a vacuum-dielectric interface," Phys. Rev. A 53. 1862-1878 (1996).

[31] E. Fucile, P. Denti, F. Borghese, R. Saija, 0. I. Sindoni, “Optical properties of a sphere in the vicinity of a plane surface," J. Opt. Soc. Am. A 14, 1505-1514 (1997).

[32] K. G. Lee, X. W. Chen, H. Eghlidi, P. Kukura, R. Lettow, A. Renn, V. Sandoghdar, and S. Götzinger, "A planar dielectric antenna for directional single-photon emission and near-unity collection efficiency," Nat. Photon. 5, 166 (2011). 